\title{
Agenesis of paranasal sinuses and nasal nitric oxide in primary ciliary dyskinesia
}

\author{
M. Pifferi*, A. Bush\#, D. Caramella`, M. Di Cicco*, M. Zangani ${ }^{\mp}$, I. Chinellato ${ }^{+}$, \\ P. Macchia* and A.L. Boner ${ }^{+}$
}

ABSTRACT: Agenesis of paranasal sinuses has only been described in case reports of patients with primary ciliary dyskinesia (PCD). As agenesis of paranasal sinuses may contribute to low nasal nitric oxide levels, a common finding in PCD, we speculated that this condition might frequently occur in PCD patients.

Patients referred for PCD evaluation were consecutively recruited for $\mathbf{3 0}$ months. In addition to standard diagnostic testing for PCD, a computed tomography (CT) scan of paranasal sinuses was performed in all subjects.

86 patients (46 children aged 8-17 yrs) were studied. PCD was diagnosed in 41 subjects and secondary ciliary dyskinesia (SCD) was diagnosed in the remaining $\mathbf{4 5}$ subjects. Frontal and/or sphenoidal sinuses were either aplastic or hypoplastic on CT scans in $30(73 \%)$ out of 41 PCD patients, but in only $17(38 \%)$ out of 45 with SCD $(p=0.002)$. There was a significant inverse correlation between the score for aplasia/hypoplasia of each paranasal sinus and nasal NO values in the PCD patients $(p=0.008, r=-0.432)$ but not in $\operatorname{SCD}(p=0.07, r=-0.271)$.

The findings of aplasia/hypoplasia of the frontal and or sphenoidal sinuses may be part of the spectrum of PCD and this finding should prompt exclusion of this condition.

KEYWORDS: Agenesis, nasal nitric oxide, paranasal sinuses, primary ciliary dyskinesia, secondary ciliary dyskinesia

$\mathrm{M}$ ost exhaled breath nitric oxide (NO) originates from the upper airways, in particular from the paranasal sinuses [1] and the nasal mucosa [2]. NO is important in local, upper airway host defense mechanisms [1]. In patients with sinusitis and those with primary ciliary dyskinesia (PCD), a disorder characterised by increased susceptibility to sinus infections, nasal NO levels are generally low $[3,4]$. To date, agenesis of paranasal sinuses has only been described in case reports of patients with PCD [5]. There is no longitudinal study in a large number of patients. We hypothesised that sinus agenesis or decreased sinus pneumatisation in PCD would be associated with a lower nasal NO and worse upper and lower airway disease. The aim of this study was to assess paranasal sinus anatomy in a large series of patients with PCD and to correlate the findings with nasal NO levels and clinical manifestations of the disease.

\section{MATERIALS AND METHODS}

\section{Subjects}

86 subjects ( 26 with situs inversus) aged $\geqslant 8$ yrs, with a clinical history and signs suggestive of PCD were consecutively evaluated in the Dept of Paediatrics at the University of Pisa (Pisa, Italy). A diagnosis of PCD was made on the basis of structural and/or functional ciliary abnormalities, as described previously [6-10]. In all subjects, ciliary motion analysis, ultrastructural assessment of cilia, ciliary function after ciliogenesis in culture of nasal brushing samples, and nasal NO measurements were performed when the subject had been free from respiratory infection for $\geqslant 4$ weeks. Other underlying conditions which could have been responsible for recurrent upper and lower respiratory tract infections were excluded. All patients underwent chest and sinus computed tomography (CT) scans, which were evaluated for the degree of pneumatisation of each paranasal sinus and the severity and extent of local inflammation.

Informed consent for nasal brushing was obtained from each adult patient or the children's parents and the local hospital ethical committee (Pisa, Italy) approved the study protocol.

\section{CT scanning of paranasal sinuses}

All patients underwent unenhanced CT scans (multislice CT; General Electric Medical Systems,
AFFILIATIONS

${ }^{*}$ Dept of Paediatrics and

"Division of Diagnostic and Interventional Radiology, University of Pisa, Pisa, and

${ }^{+}$Dept of Paediatrics, University of Verona, Verona, Italy.

\#Imperial School of Medicine, National Heart and Lung Institute, London, UK.

\section{CORRESPONDENCE}

M. Pifferi

Dept of Paediatrics

University of Pisa

Via Roma 6756126

Pisa

Italy

E-mail: m.pifferi@med.unipi.it

Received:

May 022010

Accepted after revision:

June 282010

First published online:

July 222010 
Milwaukee, MI, USA) of the sinuses and axial, sagittal and coronal images were acquired. The CT assessment of the degree of pneumatisation of each paranasal sinus was always performed by the same radiologist (D. Caramella) who was blinded to the results obtained by the other researchers. In patients aged $\geqslant 14$ yrs (when sinus development is generally complete) [11] sinus aplasia or hypoplasia were defined according to radiological criteria recently used in adults with cystic fibrosis (CF) (fig. 1, table 1) [12]. Moreover, to better evaluate the morphology of the paranasal sinuses in relation to the patients' age [11] a volumetric 3-dimensional reconstruction of the CT datasets were performed and the following scores allocated: 1 =hypoplasia; 2 =agenesis of each sinus. In consideration of the developmental stages of paranasal sinuses and in line with previous studies [11], a delay in pneumatisation in children aged $>8$ yrs was considered as hypoplasia and a total lack of pneumatisation in children aged $>10$ yrs as aplasia, even though the sphenoidal sinus only attains its mature size by the age of 14 yrs. Likewise, although the frontal sinuses achieve their final size after puberty, a delay in their pneumatisation (hypoplasia) was diagnosed either if the top of the frontal sinuses was not at the level of the orbital roof by 8 yrs of age, or if the top did not extend into the vertical portion of the frontal bone by $10 \mathrm{yrs}$ of age as previously suggested [11].

The degree of inflammation was assessed using the LundMackay system [13], in which a score of 0 is assigned to sinuses with no mucosal thickening, a score of 2 is assigned to completely opacified sinuses, and a score of 1 is assigned to
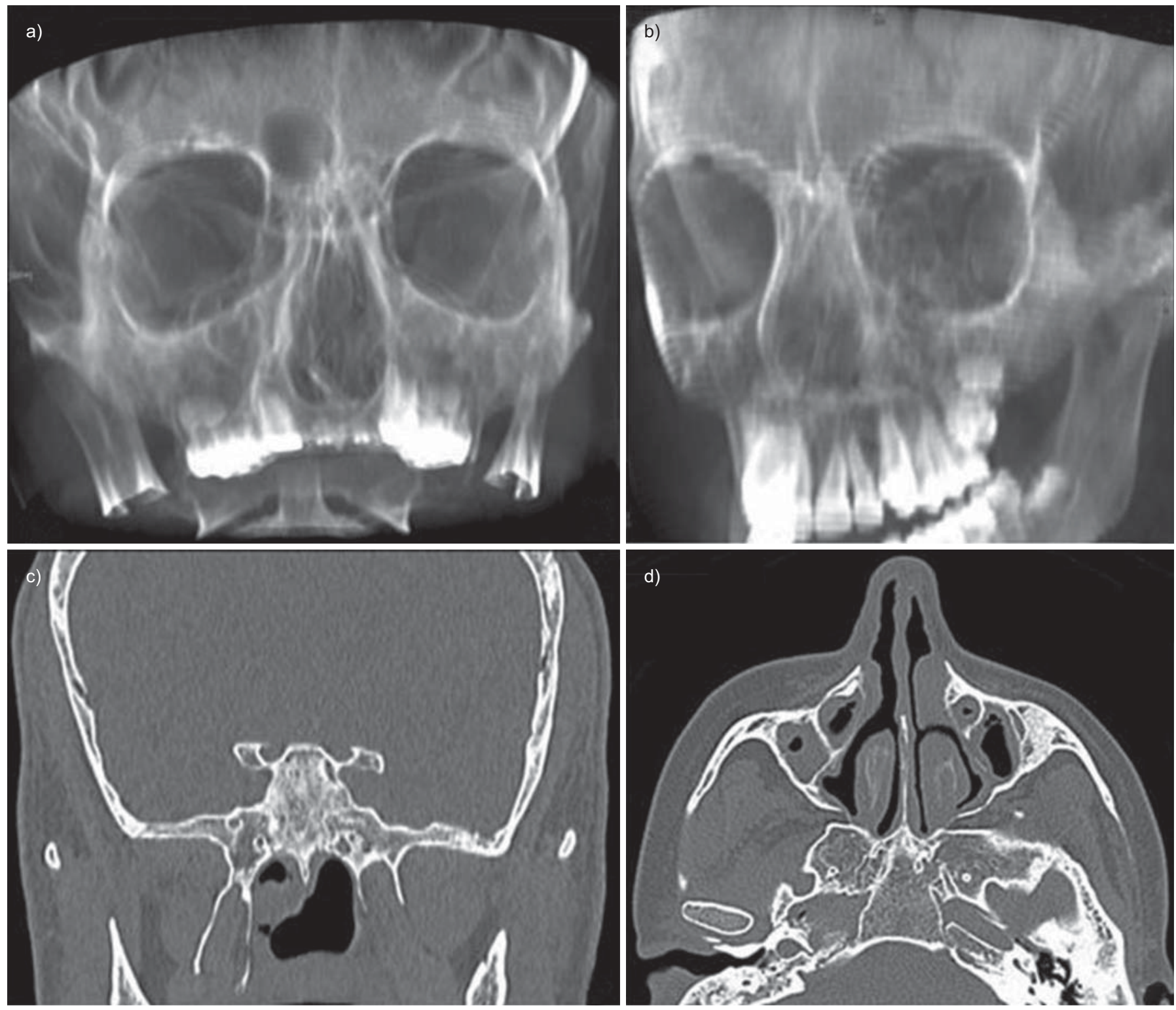

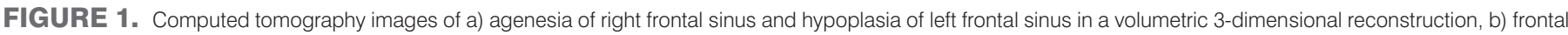

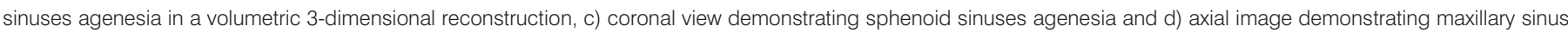
hypoplasia. 


\begin{tabular}{l}
$\begin{array}{l}\text { TABLE } 1 \text { Definitions of radiological features } \\
\text { Radiological features }\end{array}$ \\
$\begin{array}{ll}\text { Definitions } \\
\text { Frontal and sphenoid sinus aplasia } \\
\text { Frontal sinus hypoplasia }\end{array}$ \\
$\begin{array}{l}\text { Absence of bone pneumatisation } \\
\text { Oval-shaped sinus, presence of lateral margin of the sinus medially to a vertical line drawn } \\
\text { through the medial surface of the globe, and absence of intrasinus septa } \\
\text { Oval-shaped sinus, pneumatisation limited to the pre-sphenoid, anterior to the vertical plane of } \\
\text { the tuberculum sellae and absence of intrasinus septa }\end{array}$ \\
$\begin{array}{l}\text { Presence of three of the following four criteria: 1) oval-shaped sinus; 2) absence of } \\
\text { pneumatisation of the sinus below the level of the nasal floor; 3) presence of medial wall of } \\
\text { the sinus laterally to a vertical line drawn tangentially to the medial orbital border; } 4) \text { lateral } \\
\text { extension of the sinus medial to a vertical line drawn through the middle of the orbit at the level } \\
\text { of the infundibulum in the coronal plane }\end{array}$ \\
\hline
\end{tabular}

lesser degrees of abnormality. The frontal, anterior ethmoid, maxillary, posterior ethmoid and sphenoid sinuses as well as the ostiomeatal complex on each side, is scored with a possible maximum total score of 24 . As previously reported, a modification of this system was used because of the prevalence of sinus aplasia [12]. Briefly, if a patient lacked an evaluable sinus the total score was adjusted to account for the lack of this structure. This was performed by multiplying the patient's total score by the possible total 24 and dividing by the actual score for that patient (24-2 $\times$ number of missing structures) [12].

\section{CT scanning of the chest}

In all patients a high-resolution CT of the chest was performed using the same scanner. 1-mm thick slices were obtained with 10 -mm spacing $(120 \mathrm{kV}, 130 \mathrm{~mA})$ in the supine position at full inspiration. No intravenous contrast was injected and no anaesthesia was needed. All images were evaluated by the same radiologist (D. Caramella) who was blinded to the clinical data and scored using the system of BHALLA et al. [14], while bronchiectasis was identified according to standard criteria [15].

\section{Statistical analyses}

Baseline variables are described as group mean $\pm \mathrm{SD}$. Nasal NO values, scores for sinus agenesis and modified Lund-Mackay scores, which were non-normally distributed, were expressed as median (interquartile range; IQR). Differences between means were evaluated by the two-tailed un-paired t-test. Differences between medians were assessed by Mann-Whitney (Wilcoxon) U-test.

Correlations between quantitative evaluation for the degree of pneumatisation of each paranasal sinus or for the severity/ extent of local sinus inflammation and nasal NO measurements were examined using the Pearson correlation test. The same test was also applied to evaluate the correlation between sinus disease, presence of bronchiectasis and lung impairment. The Chi-squared test was used to verify the association between the complete development of paranasal sinuses and nasal NO levels $>250 \mathrm{ppb}$ in PCD patients or between their agenesis and PCD diagnosis. This value was selected because it has been previously shown to have the best combination of sensitivity $(97 \%)$ and specificity (90\%) for the diagnosis of PCD [4].
A p-value $<0.05$ was considered statistically significant. All statistical calculations were performed using SPSS version 15.0 (SPSS Inc., Chicago, IL, USA).

\section{RESULTS}

Out of the 86 (42 males) subjects studied, 46 were children (age range $8-17 \mathrm{yrs}$, mean \pm SD $10.7 \pm 2.9$ yrs) and 40 were adults (18-58 yrs, $32.0 \pm 9.2$ yrs). Immunological abnormalities were excluded in all patients. No subject had CF or CF gene mutations (the 33 most common mutations in the population were sought), and careful examination permitted the exclusion of swallowing problems and supraoesophageal complications of gastro-oesophageal reflux.

In $41(47.7 \%)$ patients (19 children) the diagnosis of PCD was established. In 30 of these (13 children) the diagnosis was made with ciliary motion analysis (abnormal motion patterns, including immotile cilia and/or very low ciliary beat frequency) and transmission electron microscopy (TEM) evaluation of cilia (alterations of the central pair and deficiencies of the dynein arms, associated with a small proportion of swollen cilia and compound cilia) while in the other 11 patients (six children) the diagnosis of PCD was reached with functional ciliary evaluation after ciliogenesis in culture when there was complete absence of either migration or rotation of spheroids. Ciliogenesis in culture was performed in patients with a highly suggestive clinical presentation but with atypical functional and structural features.

In the remaining 45 (52\%) patients (27 children), ciliary motion analysis demonstrated abnormal patterns in a small proportion of cilia, prevalence of thick cilia and low ciliary beat frequency, compatible with secondary ciliary dyskinesia (SCD). In these 45 subjects, TEM evaluation and ciliogenesis in culture confirmed the diagnosis of SCD. These subjects served as controls. There was no significant age difference between patients with PCD $(21.3 \pm 12.6 \mathrm{yrs})$ and SCD $(19.4 \pm 13.0 \mathrm{yrs})$.

Frontal and/or sphenoidal sinuses were either aplastic or hypoplastic in the CT scans of $30(73 \%)$ out of 41 PCD patients, but in only $17(38 \%)$ out of 45 with SCD (p=0.002). In the PCD patients, $26(32 \%)$ out of 82 frontal sinuses were aplastic and 17 $(21 \%)$ were hypoplastic. Likewise, 38 (46\%) out of 82 sphenoid sinuses were poorly developed, with $12(15 \%)$ being aplastic and $26(32 \%)$ being hypoplastic. Maxillary hypoplasia was less common, affecting 10 (12\%) out of 82 maxillary sinuses. 


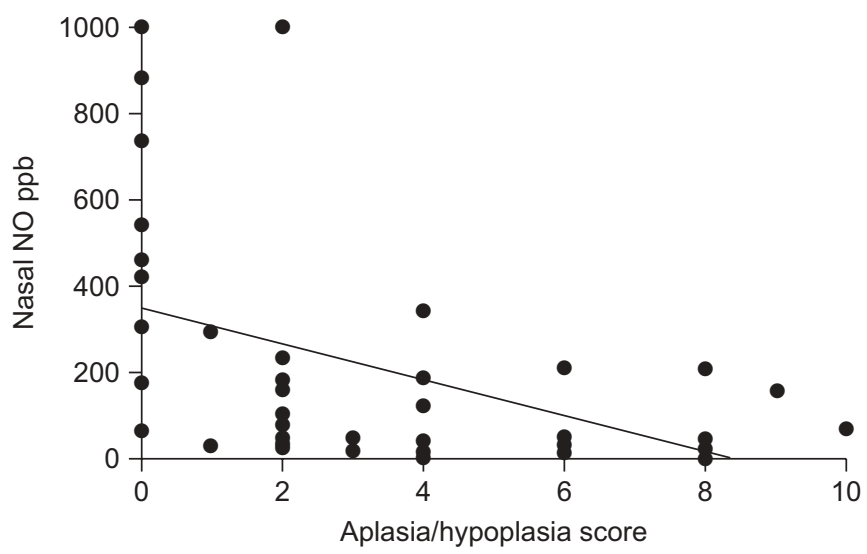

FIGURE 2. Correlation between the score for aplasia/hypoplasia of paranasal sinuses and nasal nitric oxide (NO) values in patients with primary ciliary dyskinesia. $r=-0.432, p=0.008$.

In subjects with SCD only $10(11 \%)$ out of 90 frontal sinuses were aplastic and $15(17 \%)$ were hypoplastic. Only nine (10\%) sphenoid sinuses were poorly developed, with six $(7 \%)$ being aplastic and three (3\%) hypoplastic; two $(2 \%)$ of the maxillary sinuses were hypoplastic.

There was a significant difference $(\mathrm{p}<0.0001)$ between the score for aplastic or hypoplastic sinuses in patients with PCD (median (IQR) 4.0 (6.0)) compared to subjects with SCD (0.0 (2.0)). There was no significant correlation between the age of the patients and agenesis of paranasal sinuses in either group.

In the PCD patients the group median nasal NO was $107.7 \mathrm{ppb}$ (IQR 231.4), but 10 (24\%) out of 41 had a nasal NO >250 ppb (498.2 (482.9) ppb, range 290-1,000 ppb). In the PCD group, 12 patients had specific immunoglobulin $\mathrm{E}$ to one or more perennial allergens (cat, dog, house dust mites) and/or seasonal allergens (tree, grass pollens, Parietaria officinalis) and of these only three subjects had a nasal NO level $>250 \mathrm{ppb}$ suggesting that in these patients high nasal NO is not driven by the atopic status. Three patients had nasal polyposis and all had nasal NO $<250 \mathrm{ppb}$.

The SCD group had significantly different nasal NO values (685.0 (520.2) ppb, p<0.0000001); 10 (22\%) out 45 had nasal NO $<250 \mathrm{ppb}$ (109.7 (74.1) ppb) with a range between 31 and $202 \mathrm{ppb}$. In this group 16 subjects were allergic, seven had nasal polyposis, which was associated with severe sinusitis in two subjects who also had a nasal NO level of $<250 \mathrm{ppb}$.

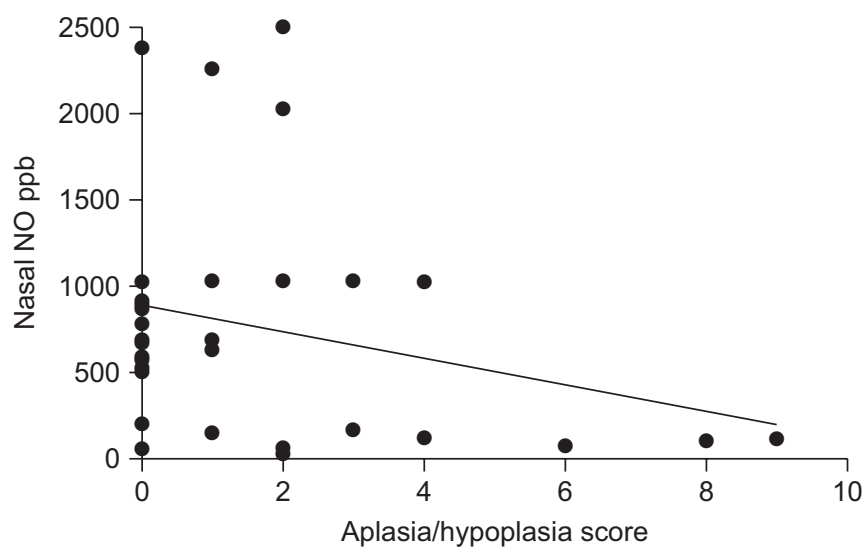

FIGURE 3. Correlation between the score for aplasia/hypoplasia of paranasa sinuses and nasal nitric oxide (NO) values in subjects with secondary ciliary dyskinesia. $r=-0.271, p=0.07$.

There was a significant inverse correlation between the score for aplasia/hypoplasia of each paranasal sinus and nasal NO values in the PCD patients ( $p=0.008, r=-0.432)$ (fig. 2$)$, but not in $\operatorname{SCD}(p=0.07, r=-0.271)$ (fig. 3$)$. There was a significant association (table 2) between the complete development of paranasal sinuses and nasal NO levels $>250 \mathrm{ppb}$ in patients with PCD $(\mathrm{p}<0.001)$. No correlation was found between aplasia/hypoplasia and bronchiectasis or lung impairment.

As expected, sinus inflammation was more frequently found in PCD patients. The median (IQR) modified Lund-Mackay score was 9.0 (6.5) in PCD, and only 3.5 (7) in subjects with SCD $(p<0.001)$. However, in both groups of patients there was a significant inverse correlation between the severity and extent of local sinus inflammation and nasal NO (PCD: $p=0.002$, $\mathrm{r}=-0.499$; SCD: $\mathrm{p}=0.01, \mathrm{r}=-0.365)$. Moreover, in PCD patients there was a significant positive correlation between agenesia/ hypoplasia of the frontal or sphenoidal sinuses and the degree of inflammation of the remaining developed sinuses $(p=0.002$, $\mathrm{r}=0.486$ ) (see supplementary material).

Finally, there was a positive correlation between sinus inflammation and the presence of bronchiectasis $(p=0.01$, $\mathrm{r}=0.362$ ) in SCD but not PCD. There was no correlation between sinus inflammation and the score of BHALLA et al. [14]. There were significant positive correlations between the score of BHALLA et al. [14] and presence of bronchiectasis in both groups (PCD: $p=0.02, r=0.363$; SCD: $p<0.001, r=0.733$ ).

\section{TABLE 2 Significant association between the complete development of paranasal sinuses and nasal nitric oxide (NO) levels $>250$ ppb in primary ciliary dyskinesia (PCD) patients}

\begin{tabular}{|c|c|c|c|}
\hline \multirow[t]{2}{*}{ Nasal NO } & \multirow[t]{2}{*}{ PCD patients } & \multicolumn{2}{|c|}{ PCD patients with score for sinuses agenesis/hypoplasia } \\
\hline & & 0 & $\geqslant 1$ \\
\hline$>250 \mathrm{ppb}$ & 10 & 7 (70) & $3(30)$ \\
\hline$<250 \mathrm{ppb}$ & 31 & $3(9.7)$ & $28(90.3)$ \\
\hline
\end{tabular}

Data are presented as $\mathrm{n}$ or $\mathrm{n}(\%)$. The significant association is $\mathrm{p}<0.001$ 


\section{DISCUSSION}

We have demonstrated that the frontal and/or sphenoidal sinuses were either aplastic or hypoplastic in a significant proportion of patients with PCD and significantly more frequently than in SCD. For ethical reasons we did not perform scans on normal controls. From the literature, the frontal sinus is the last paranasal cavity to expand and aplasia is present unilaterally in $15 \%$ and bilaterally in 5\% of normal adults [11] which is less than we observed, even in subjects with SCD. Aplasia of a sphenoidal sinus is extremely rare [11]. Therefore, a lack of pneumatisation of the sphenoidal sinus in patients aged $>10$ yrs is abnormal and PCD should be excluded. We suggest that for the first time that sinus aplasia/ hypoplasia is part of the clinical spectrum of PCD, although given the higher than normal prevalence in SCD this finding cannot be considered diagnostic. From our data, the positive predictive value (PPV) of low nasal $\mathrm{NO}$ is $75.6 \%$ for distinguishing PCD from SCD, with a negative predictive value (NPV) of $77.8 \%$. The equivalent PPV for aplasia/hypoplasia of the frontal/sphenoidal sinuses is $63.8 \%$ and the NPV is $71.8 \%$. Depending on the clinical context, possible nasal NO measurements could be used to reduce the need for sinus CT scans to diagnose sinus hypoplasia, although this would need to be confirmed on a larger series of patients. If precise anatomical information was required this could not be provided by nasal NO measurements.

The fact that only the development of those paranasal sinuses which have post-natal growth (frontal and sphenoidal) is compromised might indicate that this effect is secondary to chronic post-natal upper airways infection and inflammation $[16,17]$, and hypothetically related to the premature ossification of the cartilage surrounding the paranasal recesses $[16,17$.$] The$ higher than normal prevalence in SCD would support this hypothesis. In animal models it has been demonstrated that airfilled spaces in the cranium are formed by invasion of nasal mucous epithelium into neighbouring nasal capsule cartilages [18]. Nasal ventilation has been demonstrated to be an important factor in the development of the paranasal sinuses [19] and thus nasal obstruction, which is an early event in infants with PCD but which is also seen in SCD and other conditions [20], might be a further contributing factor to reduced expansion of the sinuses. These possibilities are further supported by the recent findings of aplastic or hypoplastic frontal and sphenoid sinuses in a high proportion of adult patients with $\mathrm{CF}$, a disease also characterised by chronic airway infection [12]. It is possible that nasal hypoventilation and chronic infection both need to be present in order to produce sinus hypoplasia, since unilateral choanal atresia is not associated with ipsilateral sinus hypoplasia [21].

Whether $\mathrm{NO}$ is an active mediator in this process or only a passive bystander is a matter of speculation. NO regulates a number of physiological processes such as blood flow, neurotransmission and host defences [22]. Genetically modified animals lacking inducible NO synthase are more vulnerable to viral and bacterial infections [23]; thus, the lower level of nasal NO usually found in PCD patients [4] could predispose to more severe upper airway infections and, we speculate, to a reduced development of the sinuses. The fact that in our patients sinus aplasia/hypoplasia was more severe in PCD than in SCD patients, that only those sinuses which develop post-natally were involved and that there was no correlation between sinuses aplasia/hypoplasia and the age of the patient suggests that both exogenous causes and genetic background contribute to pathogenesis.

As previously reported we also observed a correlation between a low level of nasal NO and the severity of sinusitis [3]. This may be due to a reduced resistance to infections [3], but the possibility that the infections may also cause a fall in nasal NO cannot be excluded in a cross-sectional study such as this. In a healthy volunteer the application of an NO synthase inhibitor topically in one maxillary sinus was associated with a unilateral marked decrease of $\mathrm{NO}$ production and with the appearance of CT proven maxillary sinusitis after 3 days [24]. Whether maxillary and/or ethmoidal sinusitis was a predisposing factor to aplasia/agenesia of post-natal developing sinuses is practically impossible to study in humans. However, the fact that a decrease of nasal NO concentration can be secondary to absence and/or occlusion of paranasal sinuses is corroborated by a study showing that baboons, which constitutively have no paranasal sinus cavities, have very low nasal NO $(\sim 1 \mathrm{ppb})$ compared to the nasal $\mathrm{NO}$ measured in rhesus monkeys with paranasal sinuses ( 100 ppb) [25].

Finally, we found a correlation between the severity of sinusitis and bronchiectasis in patients with SCD. Mucociliary clearance is an essential first-line defence of both the upper and lower airways, and it is well known that ciliary function impairment is present both in PCD and SCD. Failure to recognise and treat both upper and lower airway infections may lead to the development of chronic sinusitis as well as bronchiectasis. Chronic sinusitis and bronchiectasis are frequently associated [26], particularly when the two diseases share a common host defence abnormality [27]. In our patients with PCD the observation that there was no correlation between sinusitis and bronchiectasis is unexplained. It might possibly be related to the fact that sinusitis may be an earlier event than bronchiectasis and consequently antibiotic treatment might have lead to improvement in sinusitis severity. Since compliance with treatment decreases with age it is possible that later complications may be more severe, but this is speculative [28].

In conclusion, we suggest that the findings of aplasia/ hypoplasia of the frontal and or sphenoidal sinuses may be part of the spectrum of PCD, and this finding should prompt exclusion of this condition. However, SCD and other chronic respiratory disease can also cause sinus aplasia and hypoplasia thus specific testing for PCD should always be performed.

\section{SUPPORT STATEMENT}

Financial support was provided by Unione Industriali Pisana (Pisa, Italy).

\section{STATEMENT OF INTEREST}

None declared.

\section{REFERENCES}

1 Lundberg JO. Nitric oxide and the paranasal sinuses. Anat Rec (Hoboken) 2008; 291: 1479-1484.

2 Hood CM, Schroter RC, Doorly DJ, et al. Computational modeling of flow and gas exchange in models of the human maxillary sinus. J Appl Physiol 2009; 107: 1195-1203. 
3 Deja M, Busch T, Bachmann S, et al. Reduced nitric oxide in sinus epithelium of patients with radiologic maxillary sinusitis and sepsis. Am J Respir Crit Care Med 2003; 168: 281-286.

4 Narang I, Ersu R, Wilson NM, et al. Nitric oxide in chronic airway inflammation in children: diagnostic use and pathophysiological significance. Thorax 2002; 57: 586-589.

5 Gómez R, Pérez Trullen A, Ruiz C, et al. Primary ciliary dyskinesia with frontal sinus agenesis. Acta Otorrinolaringol Esp 1997; 48: 315-316.

6 Rutland J, Cole PJ. Non-invasive sampling of nasal cilia for measurement of beat frequency and study of ultrastructure. Lancet 1980; 2: 564-565.

7 Pifferi M, Cangiotti AM, Ragazzo V, et al. Primary ciliary dyskinesia: diagnosis in children with inconclusive ultrastructural evaluation. Pediatr Allergy Immunol 2001; 12: 274-282.

8 Carlén B, Stenram U. Ultrastructural diagnosis in the immotile cilia syndrome. Ultrastruct Pathol 1987; 11: 653-658.

9 Pifferi M, Montemurro F, Cangiotti AM, et al. Simplified cell culture method for the diagnosis of atypical primary ciliary dyskinesia. Thorax 2009; 64: 1077-1081.

10 Baraldi E, de Jongste JC, Gatson B, et al. Measurement of exhaled nitric oxide in children, 2001. Eur Respir J 2002; 20: 223-237.

11 Scuderi AJ, Harnsberger HR, Boyer RS. Pneumatization of the paranasal sinuses: normal features of importance to the accurate interpretation of CT scans and MR images. AJR Am J Roentgenol 1993; 160: 1101-1104.

12 Orlandi RR, Wiggins RH 3rd. Radiological sinonasal findings in adults with cystic fibrosis. Am J Rhinol Allergy 2009; 23: 307-311.

13 Hopkins C, Browne JP, Slack R, et al. The Lund-Mackay staging system for chronic rhinosinusitis: how is it used and what does it predict? Otolaryngol Head Neck Surg 2007; 137: 555-561.

14 Bhalla M, Turcios N, Aponte V, et al. Cystic fibrosis: scoring system with thin-section CT. Radiology 1991; 179: 783-788.

15 Pifferi M, Caramella D, Bulleri A, et al. Pediatric bronchiectasis: correlation of HRCT, ventilation and perfusion scintigraphy, and pulmonary function testing. Pediatr Pulmonol 2004; 38: 298-303.
16 Sato I, Sunohara M, Mikami A, et al. Immunocytochemical study of the maxilla and maxillary sinus during human fetal development. Okajimas Folia Anat Jpn 1998; 75: 205-216.

17 Ellington JK, Elhofy A, Bost KL, et al. Involvement of mitogenactivated protein kinase pathways in Staphylococcus aureus invasion of normal osteoblasts. Infect Immun 2001; 69: 5235-5242.

18 Smith TD, Rossie JB, Docherty BA, et al. Fate of the nasal capsular cartilages in prenatal and perinatal tamarins (Saguinus geoffroyi) and extent of secondary pneumatization of maxillary and frontal sinuses. Anat Rec (Hoboken) 2008; 291: 1397-1413.

19 Freng A. Congenital choanal atresia. Scand J Plast Reconstr Surg 1978; 12: 261-265.

20 Bush A, Chodhari R, Collins N, et al. Primary ciliary dyskinesia: current state of the art. Arch Dis Child 2007; 92: 1136-1140.

21 Guimarães RE, Dos Anjos GC, Becker CG, et al. Absence of nasal air flow and maxillary sinus development. Braz J Otorhinolaryngol 2007; 73: 161-164.

22 Moncada S, Higgs A. The L-arginine-nitric oxide pathway. New Engl J Med 1993; 329: 2002-2012.

23 Wei XQ, Charles IG, Smith A, et al. Altered immune responses in mice lacking inducible nitric oxide synthase. Nature 1995; 375: 408-411.

24 Lundberg JO. Acute purulent sinusitis triggered by topical nasal nitric oxide synthase inhibition. Am J Respir Crit Care Med 2005; 172: 512-513.

25 Lewandowski K, Busch T, Lohbrunner $\mathrm{H}$, et al. Low nitric oxide concentrations in exhaled gas and nasal airways of mammals without paranasal sinuses. J Appl Physiol 1998; 85: 405-410.

26 Shoemark A, Ozerovitch L, Wilson R. Aetiology in adult patients with bronchiectasis. Respir Med 2007; 101: 1163-1170.

27 Loebinger MR, Bilton D, Wilson R. Upper airway 2: bronchiectasis, cystic fibrosis and sinusitis. Thorax 2009; 64: 1096-1101.

28 Pifferi M, Bush A, Di Cicco M, et al. Health-related quality of life and unmet needs in patients with primary ciliary dyskinesia. Eur Respir J 2010; 35: 787-794. 